\title{
Pattern of lipid profile in patients attending metabolic clinic in Oghara, Delta State, Nigeria
}

\author{
Voke Peter Orugbo \\ Department of Chemical Pathology, Delta State University, Abraka, Delta State, Nigeria
}

\begin{abstract}
Owing to its high prevalence, dyslipidemia is rapidly becoming a major public heart issue worldwide, and especially in Nigeria. Although it is a preventable significant risk factor for coronary heart disease, it is a common leading cause of death in Nigeria. The study therefore investigated the lipid profiles of patients attending Metabolic Clinic at the Delta State University Teaching Hospital (DELSUTH). A total of 713 participants were recruited for the study. Blood samples $(5 \mathrm{mls})$ were collected via venipuncture from each of the participants and distributed into tubes containing EDTA and fluoride oxalate. A spectrophotometer was used to conduct the lipid analysis, and the normal operating assay protocol was followed. Results showed total cholesterol in the male $(194.6 \mathrm{mg} / \mathrm{dL})$ were generally lower than in the females, particularly for participants below the age of 40 . However, as the
\end{abstract}

Correspondence: Voke Peter Orugbo, Department of Chemical Pathology, Delta State University, PMB 1, Abraka, Delta State, Nigeria. E-mail: drorugbopeter@gmail.com

Key words: Dyslipidemia; hypercholesterolemia; total cholesterol; HDL; LDL; antherogenic ratio; Castelli ratios.

Acknowledgements: The authors are grateful to Miss Loveth Erhijodo, for SPSS data entering.

Conflict of interest: The author declares no conflict of interest.

Availability of data and materials: All data generated or analyzed during this study are included in this published article.

Ethics approval and consent to participate: All procedures performed in studies involving human participants were in accordance with the ethical standards of the Institutional Research Committee and with the 1964 Helsinki declaration and its latest amendment. Patients' consent was obtain prior to recruiting.

Consent for publication: Written informed consent was obtained from a legally authorized representative(s) for anonymized patient information to be published in this article.

Received for publication: 5 March 2021

Revision received: 27 March 2021

Accepted for publication: 2 April 2021.

This work is licensed under a Creative Commons Attribution NonCommercial 4.0 License (CC BY-NC 4.0).

${ }^{\circ}$ Copyright: the Author(s), 2021

Licensee PAGEPress, Italy

Annals of Clinical and Biomedical Research 2021;2:139

doi:10.4081/acbr.2021.139 ages progressed (that is, above 40 years), total cholesterol in males became higher than those in the females. Antherogenic ratio as well as antherogenic index of plasma were higher in the female gender at ages below 40 years. The study showed that the risk of hypercholesterolemia may be higher within the active age period of 30-60 years. As seen in the current study, plasma lipid levels change drastically by sexual development or maturity, and the trends vary by age and sex. The study also significantly demonstrated the elevated lipids levels in younger women in the study population than older men. When assessing screening and diagnostic criteria for classifying individuals with elevated blood lipid levels, pubertal or sexual growth may be taken into account.

\section{Introduction}

Lipid metabolism, including phospholipids and cholesterol for cell membrane formation, offers important building blocks for cell proliferation. Since lipids are insoluble in plasma, they are bound to lipoproteins and transferred to different tissues, where they are used for a number of functions such as energy metabolism, steroid hormone synthesis, and bile acid formation. Lipids have many functions in the body, including serving as chemical messengers, energy storage and delivery. A variety of factors that activate sterol regulatory element binding proteins may Stimulate Intracellular Lipid Synthesis (SREBP). As part of the lipoprotein particles, fats such as cholesterol and triglycerides are taken through the bloodstream. It has consistently been shown that serum cholesterol (and therefore LDL cholesterol) is a significant risk factor for Cardiovascular Disease (CVD) and other major cardiovascular diseases. $^{1-5}$

Owing to its high prevalence, dyslipidemia is rapidly becoming a major public heart issue worldwide, and especially in Nigeria. Although it is a preventable significant risk factor for coronary heart disease, it is a common leading cause of death in Nigeria. ${ }^{2-4}$ The relationship is continuous, graded, solid, predictive, independent of other risk factors and generally assessed as linked to causation. Where other risk factors are present concurrently, the effect of cholesterol on the risk of CVD is increased. Cholesterol is a risk factor for getting the first heart attack. It tends to be very effective in predicting a repeated occurrence. .-8 $^{6-8}$

CVDs are the leading cause of death worldwide, with rates of CVD-related death increasing rapidly in Nigeria $^{2}$ and other lowand middle-income countries. Nigeria can be assumed to have been in the first stage in the previous 50 years, as much of the cardiovascular disease presentations were attributed to malnutrition and communicable diseases. The most popular CVDs at the time were rheumatic heart disease and cardiomyopathies; these are significantly linked to dyslipidemia. ${ }^{2}$ Lipid levels have historically been documented to be a risk factor for CVD in patients, but the degree to which this association between lipid levels and CVD exists varies from person to person. ${ }^{1,3,5,7,8}$ Therefore, the purpose of the study is to present the lipid profile of patients attending 
Table 1. Clinical presentation of participants.

DELSUTH, Oghara Metabolic Clinic, with a view to determining possibility of linking it with risk of cardiovascular impairments.

\section{Materials and Methods}

In this study, which is a cross-sectional study, a total of 713 participants were recruited. There were those who registered to DELSUTH's Metabolic Clinic for routine check-ups or on appointment. The Delta State University Teaching Hospital (DELSUTH) is an affiliate of the Delta State University (Abraka). With provision to provide medical care in over 20 Medical Specialties, the hospital attends to patients all over the entire Delta State and neighbouring States.

Informed consent was obtained from each recruited study participant before clinical presentations and other relevant details were obtained and subsequently segregated according to certain age groups, as well as gender.

Venipuncture was performed such that approximately $5 \mathrm{mls}$ of blood was obtained from each of the participants and distributed into tubes containing EDTA and fluoride oxalate. The entire blood was centrifuged, and the plasma was collected into $5 \mathrm{~mL}$ plain tubes with a Pasteur pipette and processed at $-20^{\circ} \mathrm{C}$ before lipid analysis was performed. A spectrophotometer was used to conduct the lipid analysis, and the normal operating assay protocol was followed. The following equations, as used in Idemudia and Atoe, ${ }^{3}$ were used to measure the blood lipid indexes.

Friedewald equation for LDL Chol. $=\mathrm{TC}-\mathrm{TG} / 5-\mathrm{HDL}$ Chol. $(\mathrm{mg} / \mathrm{dL})$

Non-HDL cholesterol, NHDL $=$ Total Chol. - HDL Chol

Very low density lipoprotein, $\mathrm{VLDL}=$ Triglycerides $/ 5$

Atherogenic index of plasma, AIP $=\mathrm{Log}$ (TG/HDLChol.)

Atherogenic coefficient, AC $=$ Non HDL Chol. / HDL Chol.

Castelli Risk Index I = TC / HDL Chol.

Castelli Risk Index II = LDLChol./ HDLChol.

Statistical analyses of the data obtained in the analysis were performed using SPSS ${ }^{\circ}$ version-21. When required, the results were presented as 95 th percentiles as well as the mean of replicated data.

\section{Results}

Clinical presentation of the study participants showed that out of 713 assessed, 24\% were sufferers of hypertension (Table 1). However, $18.5 \%$ were on routine checkup. According to age distribution, most of the study participants were with the 51-60 years category (29.9\%). Mean distribution of lipid profiles among the study population according to gender has been presented (Table 2). Total cholesterol ranged 119.63-125.94 mg/dL ( $>>0.05$ ). HDLCholesterol levels in female $(36.59 \mathrm{mg} / \mathrm{dL})$ was significantly higher than those of the male $(33.31 \mathrm{mg} / \mathrm{dL})(\mathrm{p}<0.05)$. Antherogenic ratio (AR) ranged from 5.76-5.85, whereas antherogenic index plasma did not significantly differ among the gender (0.51). Differences in Castelli Ratio (I) in both male (6.76) and female (6.85) were minimal ( $>>0.05$ ) (Table 2).

The $95^{\text {th }}$ percentile of lipid profiles among the study population has been presented (Table 3). Total cholesterol in the male was $194.6 \mathrm{mg} / \mathrm{dL}$ at $11-20$ years compared to $209.5 \mathrm{mg} / \mathrm{dL}$ in the females within similar age category. Similarly, in the 21-30 years age category, total cholesterol in the female $(230.6 \mathrm{mg} / \mathrm{dL})$ was higher than those of the male $(226.6 \mathrm{mg} / \mathrm{dL})$. However, as the ages progressed (that is, above 40 years), total cholesterol in males

\begin{tabular}{lc} 
Clinical condition & n $(\%)$ \\
Acute decompensation & $4(0.6)$ \\
Acute left ventricular failure & $23(3.2)$ \\
\hline Cervical myelopathy & $7(1.0)$ \\
CCF 2^O HHDX & $39(5.5)$ \\
\hline Chronic left leg ulcer & $1(0.1)$ \\
Chronic Liver dx & $35(4.9)$ \\
\hline CKD & $13(1.8)$ \\
CVA & $33(4.6)$ \\
\hline CVD & $37(5.2)$ \\
Decompensated HHF & $4(0.6)$ \\
\hline DKA & $2(0.3)$ \\
Elevated BP & $13(1.8)$ \\
\hline FSLP & $7(1.0)$ \\
Hemorrhage CVD & $2(0.3)$ \\
\hline Hepatomegaly & $2(0.2)$ \\
HHDx & $33(4.6)$ \\
\hline HIV Encephalopathy & $17(2.4)$ \\
Hypertension & $171(24.0)$ \\
\hline Hypercholectera & $1(0.1)$ \\
Left foot gangrene 2^0 peripheral & $5(0.7)$ \\
\hline Left hemispheric & $2(0.3)$ \\
Left hypertrophy & $2(0.3)$ \\
\hline Lucipunt wart failure & $3(0.4)$ \\
Multiple myeloma & $12(1.7)$ \\
\hline P.VHD & $13(1.8)$ \\
\hline Palpitation IRC & $11(1.5)$ \\
\hline Poorly controlled BP & $19(2.7)$ \\
PUD & $1(0.1)$ \\
\hline Recurrent miscarriage & $2(0.3)$ \\
Routine checkup & $132(18.5)$ \\
\hline RVD & $7(1.0)$ \\
Seizure & $7(1.0)$ \\
\hline Somatization disorder & $3(0.4)$ \\
\hline Stroke DM & $22(3.1)$ \\
\hline
\end{tabular}

Table 2. Mean distribution of lipid profiles among the study population according to gender.

\begin{tabular}{lcccc} 
Parameters & $\begin{array}{c}\text { Male } \\
(\mathrm{n}=356)\end{array}$ & $\begin{array}{c}\text { Female } \\
(\mathrm{n}=357)\end{array}$ & T-test & P-value \\
TC $(\mathrm{mg} / \mathrm{dL})$ & 119.63 & 125.94 & 1.699 & 0.090 \\
HDL $(\mathrm{mg} / \mathrm{dL})$ & 33.31 & 36.59 & 2.500 & $0.013^{*}$ \\
\hline TRIG $(\mathrm{mg} / \mathrm{dL})$ & 88.44 & 90.10 & 0.362 & 0.718 \\
LDL $(\mathrm{mg} / \mathrm{dL})$ & 68.63 & 71.33 & 0.732 & 0.464 \\
\hline VLDL $(\mathrm{mg} / \mathrm{dL})$ & 17.69 & 18.02 & 0.362 & 0.718 \\
NHDL $(\mathrm{mg} / \mathrm{dL})$ & 86.32 & 89.35 & 0.782 & 0.435 \\
\hline AR & 5.76 & 5.85 & 0.080 & 0.936 \\
AIP & 0.51 & 0.51 & 0.031 & 0.975 \\
\hline CRI (I) & 6.76 & 6.85 & 0.080 & 0.936 \\
CRI (II) & 4.73 & 4.67 & -0.062 & 0.951 \\
\hline
\end{tabular}

TC: total cholesterol; HDL: HDL-cholesterol; LDL: LDL—cholesterol; TRIG: triglycerides; VLDL: Very Low Density Cholesterol: NHDL: Non-HDL—cholesterol; AR: antherogenic ratio; AIP: Antherogenic Index of Plasma; CRI (I\&II): Castelli Ratio (I\&II). 
became higher than those in the female. Antherogenic ratio as well as antherogenic index of plasma were higher in the female gender at ages below 40 years (Table 3 ).

For the difficulty of obtaining $95^{\text {th }}$ percentile of older ages due to low number, the means of the profiles within these age categories were obtained. Results showed significant differences in mean values for TC and LDL respectively (Table 4). Whereas, TC was highest in the $>100$ years age category $(=151.67 \mathrm{mg} / \mathrm{mL})$, the lowest mean TC value was obtained in the 71-80 years category (= $100.08 \mathrm{mg} / \mathrm{mL}$ ). No significant differences in lipid indices and rations were obtained among the age categories.

Mean values for lipid profiles according to the clinical presentations (irrespective of gender and age category) of the participants showed that significant distribution of the lipid ratios (AR, AIP, CRI I and CRI II) respectively (Table 5). The mean TC was highest in participants who were sufferers of Hemorrhagic stroke (159.0 $\mathrm{mg} / \mathrm{mL}$ ) and Lucipurr heart failure $(137.3 \mathrm{mg} / \mathrm{mL})$ respectively. TC of $124.9 \mathrm{mg} / \mathrm{mL}$ was recorded for those on routine checkup.

In an attempt to know number of participants based on their respective clinical presentations that were positive for hypercholesterolemia, data on clinical details of the participants were cross-tabulated against occurrence of hypercholesterolemia based on a cholesterol reference level of $92-180 \mathrm{mg} / \mathrm{dL}$ (Table 6). Out of the 713 participants, a total of 113 were positive for hypercholesterolemia, representing $15.84 \%$ of the study population; $20.4 \%$ of the total population of participants that were positive for hypercholesterolemia only reported to the clinic on routine check-up, whereas $30.1 \%$ had hypertension. However, according to age categorization, hypercholesterolemia was more prominent within the 41-60 years bracket (23.9-25.7\%; Table 7) and least above the age of sixty.

\section{Discussion}

The current study examines the lipid profile of patients at the DELSUTH Delta State Metabolic Clinic. The female gender had higher total cholesterol (as determined by the 95 th percentile) than the male gender when both were over 40 years old. The effects of the lipid ratios were the same. Until midlife (ages 50 to 60), women have a lower risk of atherosclerotic cardiovascular disease than men, though the difference starts to close after menopause. Swiger et al. ${ }^{9}$ earlier hypothesized that the average lipid profile of women undergoes unfavorable changes compared with men after midlife. Godsland et al. ${ }^{10}$ opined that as women age, the serum concentrations of triglycerides, low-density lipoprotein cholesterol and Total Cholesterol (TC) surpass those in men. Menopause itself is associated with an increased prevalence of dyslipidemia but not hypertension or insulin resistance, independent of the effect of chronological aging. ${ }^{11-13}$

Table 3. Presentation lipid profiles $\left(95^{\text {th }}\right.$ percentiles) among the study population according to gender and age.

\begin{tabular}{|c|c|c|c|c|c|c|c|c|c|c|c|}
\hline Age (years) & (n) & $\begin{array}{c}\mathrm{TC} \\
(\mathrm{mg} / \mathrm{dL})\end{array}$ & HDL & TRI & LDL & VLDL & NHDL & AR & AIP & CRI_I & CRI_II \\
\hline \multicolumn{12}{|l|}{ Male } \\
\hline$<11$ & 1 & NA & NA & $\mathrm{NA}$ & NA & NA & NA & $\mathrm{NA}$ & NA & NA & NA \\
\hline $11-20$ & 23 & 195 & 56 & 228 & 147 & 45.6 & 154 & 8.4 & 0.9 & 9.4 & 7.2 \\
\hline $21-30$ & 53 & 227 & 75.4 & 216 & 179 & 43.2 & 192 & 31.8 & 1.3 & 32.8 & 28.5 \\
\hline $31-40$ & 62 & 211 & 52.9 & 226 & 149 & 45.3 & 194 & 22.1 & 1.4 & 23.1 & 16.7 \\
\hline $41-50$ & 69 & 227 & 69.5 & 192 & 181 & 38.3 & 214 & 23.9 & 1.4 & 24.9 & 20.5 \\
\hline $51-60$ & 109 & 197 & 64 & 194 & 147 & 38.7 & 163 & 8.9 & 1.1 & 9.9 & 7.8 \\
\hline $61-70$ & 23 & 245 & 61.6 & 220 & 190 & 44 & 209 & 28.7 & 1.5 & 29.7 & 22.8 \\
\hline $71-80$ & 5 & NA & $\mathrm{NA}$ & NA & NA & NA & NA & $\mathrm{NA}$ & NA & NA & NA \\
\hline $81-90$ & 1 & NA & $\mathrm{NA}$ & $\mathrm{NA}$ & NA & $\mathrm{NA}$ & NA & $\mathrm{NA}$ & NA & NA & NA \\
\hline $91-100$ & 1 & NA & $\mathrm{NA}$ & NA & NA & $\mathrm{NA}$ & NA & NA & NA & NA & NA \\
\hline$>100$ & 0 & $\mathrm{NA}$ & $\mathrm{NA}$ & $\mathrm{NA}$ & $\mathrm{NA}$ & $\mathrm{NA}$ & $\mathrm{NA}$ & $\mathrm{NA}$ & NA & $\mathrm{NA}$ & NA \\
\hline \multicolumn{12}{|l|}{ Female } \\
\hline$<11$ & 6 & 227 & 72 & 99 & 185 & 19.8 & 205 & 10.8 & 1 & 11.8 & 9.8 \\
\hline $11-20$ & 25 & 210 & 66.7 & 202 & 172 & 40.3 & 204 & 119 & 2.1 & 120 & 91.4 \\
\hline $21-30$ & 52 & 231 & 73.7 & 241 & 148 & 48.3 & 190 & 84.4 & 1.9 & 85.4 & 68.6 \\
\hline $31-40$ & 76 & 225 & 68.8 & 212 & 187 & 42.4 & 201 & 18 & 1.2 & 19 & 15.5 \\
\hline $41-50$ & 64 & 234 & 71.5 & 213 & 189 & 42.5 & 204 & 17.6 & 0.9 & 18.6 & 16.4 \\
\hline $51-60$ & 104 & 231 & 65 & 216 & 176 & 43.2 & 187 & 10.7 & 1.4 & 11.7 & 10.1 \\
\hline $61-70$ & 7 & $\mathrm{NA}$ & $\mathrm{NA}$ & $\mathrm{NA}$ & NA & $\mathrm{NA}$ & NA & $\mathrm{NA}$ & NA & NA & NA \\
\hline $71-80$ & 13 & NA & NA & $\mathrm{NA}$ & NA & NA & $\mathrm{NA}$ & $\mathrm{NA}$ & $\mathrm{NA}$ & $\mathrm{NA}$ & $\mathrm{NA}$ \\
\hline $81-90$ & 3 & NA & NA & $\mathrm{NA}$ & $\mathrm{NA}$ & NA & NA & $\mathrm{NA}$ & NA & NA & NA \\
\hline $91-100$ & 3 & NA & NA & NA & NA & NA & NA & $\mathrm{NA}$ & NA & NA & NA \\
\hline$\geq 100$ & 3 & NA & $\mathrm{NA}$ & $\mathrm{NA}$ & NA & $\mathrm{NA}$ & NA & $\mathrm{NA}$ & NA & NA & NA \\
\hline
\end{tabular}

TC: total cholesterol; HDL: HDL-cholesterol; LDL: LDL—cholesterol; TRIG: triglycerides; VLDL: Very Low Density Cholesterol; NHDL: Non-HDL—cholesterol: AR: antherogenic ratio; AIP: Antherogenic Index of Plasma; CRI (I\&II): Castelli Ratio (I\&II); Palpitation IRC: Palpitation Irrespective of Underlying Cause. 
Table 4. Mean distribution of lipid profiles among the study population according to age.

\begin{tabular}{|c|c|c|c|c|c|c|c|c|c|c|c|}
\hline Age (years) & (n) & $\begin{array}{c}\mathrm{TC} \\
\text { (mg/dL) }\end{array}$ & HDL & TRI & LDL & VLDL & NHDL & $\mathbf{A R}$ & AIP & CRI_I & CRI_II \\
\hline$<11$ & 17 & 137.47ab & 34.53 & 83.68 & $96.29 \mathrm{ab}$ & 102.9 & 19.26 & 3.53 & 0.51 & 4.53 & 2.88 \\
\hline $11-20$ & 48 & 115.62ab & 32.98 & 64.88 & 88.85ab & 82.65 & 17.77 & 8.21 & 0.55 & 9.21 & 6.62 \\
\hline $21-30$ & 105 & 124.54ab & 35.41 & 69.29 & 99.21ab & 89.13 & 19.84 & 7.97 & 0.56 & 8.97 & 6.28 \\
\hline $31-40$ & 138 & 121.28ab & 34.8 & 68.67 & 89.04ab & 86.48 & 17.81 & 5.57 & 0.52 & 6.57 & 4.29 \\
\hline $41-50$ & 133 & $127.8 \mathrm{ab}$ & 35.66 & 73.41 & $93.63 \mathrm{ab}$ & 92.14 & 18.73 & 6.85 & 0.52 & 7.85 & 5.69 \\
\hline $51-60$ & 213 & 119.47ab & 35.36 & 67.44 & 83.36ab & 84.11 & 16.67 & 4.14 & 0.49 & 5.14 & 3.43 \\
\hline $61-70$ & 36 & 133.86ab & 32.64 & 84.33 & $84.47 a b$ & 101.2 & 16.89 & 6.22 & 0.51 & 7.22 & 5.4 \\
\hline $71-80$ & 12 & $100.08 \mathrm{a}$ & 31.67 & 54.87 & 67.75ab & 68.42 & 13.55 & 2.06 & 0.29 & 3.06 & 1.6 \\
\hline $81-90$ & 4 & 123.50ab & 35 & 70.75 & $88.75 \mathrm{ab}$ & 88.5 & 17.75 & 3.82 & 0.38 & 4.82 & 3.33 \\
\hline $91-100$ & 4 & $106.25 \mathrm{ab}$ & 36.5 & 47.55 & $111.00 \mathrm{a}$ & 69.75 & 22.2 & 2.33 & 0.64 & 3.33 & 1.52 \\
\hline$>100$ & 3 & $151.67 \mathrm{~b}$ & 36.67 & 103 & $60.00 \mathrm{~b}$ & 115 & 12 & 4.23 & 0.48 & 5.23 & 3.8 \\
\hline F-statistic & & 1.083 & 0.21 & 0.957 & 0.851 & 0.955 & 0.851 & 0.702 & 0.773 & 0.702 & 0.645 \\
\hline p-value & & 0.373 & 0.995 & 0.48 & 0.58 & 0.482 & 0.58 & 0.723 & 0.655 & 0.723 & 0.775 \\
\hline Sign. & & $*$ & NS & NS & * & NS & NS & NS & NS & NS & NS \\
\hline
\end{tabular}

Means on the same column with similar alphabets do not differ from each other significantly (p>0.05). TC: total cholesterol; HDL: HDL-cholesterol; LDL: LDL—cholesterol; TRIG: triglycerides; VLDL: Very Low Density Cholesterol; NHDL: Non-HDL—cholesterol: AR: antherogenic ratio; AIP: Antherogenic Index of Plasma; CRI (I\&II): Castelli Ratio (I\&II); Palpitation IRC: Palpitation Irrespective of Underlying Cause.

Table 5. Mean distribution of lipid profiles among the study population according to clinical presentation.

\begin{tabular}{|c|c|c|c|c|c|c|c|c|c|c|}
\hline Clinical condition & $\mathrm{TC}$ & HDL & TRI & LDL & VLDL & NHDL & AR & AIP & CRI_I & CRI_II \\
\hline Acute left Ventricular failure & 122 & 40.5 & 87.4 & 63.6 & 17.5 & 81.1 & 3 & 0.4 & 4 & 2.4 \\
\hline Routine checkup & 125 & 33.9 & 86.1 & 73.8 & 17.2 & 91 & 5.3 & 0.5 & 6.3 & 4.3 \\
\hline CVA & 121 & 35.5 & 88 & 68 & 17.6 & 85.6 & 3.3 & 0.5 & 4.3 & 2.7 \\
\hline HTN & 128 & 35 & 91 & 74.5 & 18.2 & 92.7 & 5.6 & 0.5 & 6.6 & 4.5 \\
\hline$\overline{\mathrm{CCF}} 2^{\wedge} \mathrm{O}$ HHDX & 132 & 33.8 & 83.8 & 81.4 & 16.8 & 98.2 & 5.9 & 0.5 & 6.9 & 4.8 \\
\hline T2 DM & 112 & 35.1 & 100 & 57.2 & 20 & 77.3 & 11.6 & 0.6 & 12.6 & 9 \\
\hline HHDx & 113 & 32.9 & 84 & 63.7 & 16.8 & 80.5 & 3.4 & 0.4 & 4.4 & 2.8 \\
\hline HIV Encephalopathy & 108 & 42.5 & 71.5 & 51 & 14.3 & 65.3 & 2.1 & 0.4 & 3.1 & 1.7 \\
\hline Stroke & 122 & 35.8 & 90 & 68.6 & 18 & 86.6 & 4.1 & 0.5 & 5.1 & 3.5 \\
\hline Seizure & 133 & 21.4 & 105 & 90.9 & 20.9 & 112 & 35.9 & 0.9 & 36.9 & 31.8 \\
\hline Chronic Liver $\mathrm{dx}$ & 113 & 33.3 & 104 & 59.2 & 20.7 & 79.9 & 6.2 & 0.6 & 7.2 & 4.6 \\
\hline RVD & 127 & 38.6 & 99.4 & 69 & 19.9 & 88.9 & 10.5 & 0.4 & 11.5 & 9.8 \\
\hline PUD & 100 & 29 & 9 & 69.2 & 1.8 & 71 & 2.5 & 0.5 & 3.5 & 2.4 \\
\hline CVD & 115 & 37 & 90.4 & 60.4 & 18.1 & 78.5 & 3.2 & 0.4 & 4.2 & 2.6 \\
\hline CKD & 95.6 & 27.7 & 67.6 & 54.4 & 13.5 & 67.9 & 11.4 & 0.5 & 12.4 & 10.7 \\
\hline Multiple myeloma & 101 & 41.3 & 98.9 & 39.8 & 19.8 & 59.6 & 8.1 & 0.6 & 9.1 & 6.4 \\
\hline Cervical myelopathy & 116 & 44.6 & 108 & 49.5 & 21.6 & 71.1 & 4.9 & 0.7 & 5.9 & 3.6 \\
\hline Acute decompensation & 115 & 31.3 & 104 & 62.4 & 20.9 & 83.3 & 10.8 & 0.7 & 11.8 & 8.7 \\
\hline Lucipunt wart failure & 137 & 38 & 42.3 & 90.9 & 8.5 & 99.3 & 2.5 & 0.2 & 3.5 & 2.3 \\
\hline DKA & 72.5 & 27.5 & 85.5 & 27.9 & 17.1 & 45 & 1.8 & 1.3 & 2.8 & 0.8 \\
\hline Poorly controlled BP & 142 & 36.3 & 96.7 & 86 & 19.3 & 105 & 4.2 & 0.5 & 5.2 & 3.5 \\
\hline P.VHD & 118 & 39.5 & 88.2 & 61.3 & 17.6 & 78.9 & 2.5 & 0.3 & 3.5 & 2.1 \\
\hline FSLP & 116 & 33.3 & 78.4 & 66.7 & 15.7 & 82.4 & 17.4 & 0.6 & 18.4 & 13.9 \\
\hline Left foot gangrene $2^{\wedge} 0$ peripheral & 149 & 30.2 & 56.4 & 108 & 11.3 & 119 & 5.2 & 0.6 & 6.2 & 4.9 \\
\hline Recurrent miscarriage & 81 & 46.5 & 62.5 & 22 & 12.5 & 34.5 & 0.8 & 0.4 & 1.8 & 0.5 \\
\hline Hemorrhage CVD & 159 & 37.5 & 123 & 96.9 & 24.6 & 122 & 3.1 & 0.4 & 4.1 & 2.4 \\
\hline Somatization disorder & 123 & 35.7 & 61.3 & 74.7 & 12.3 & 87 & 2.9 & 0.4 & 3.9 & 2.5 \\
\hline Elevated BP & 131 & 38 & 86.2 & 76.1 & 17.2 & 93.4 & 3.1 & 0.6 & 4.1 & 2.5 \\
\hline Palpitation IRC & 135 & 34 & 100 & 80.8 & 20 & 101 & 4.6 & 0.5 & 5.6 & 3.8 \\
\hline Hypercholectera & 86 & 1 & 202 & 44.6 & 40.4 & 85 & 85 & 2.3 & 86 & 44.6 \\
\hline Decompensated HHF & 126 & 37 & 99.5 & 69.4 & 19.9 & 89.3 & 2.8 & 0.4 & 3.8 & 2.2 \\
\hline Left hemispheric & 207 & 17.5 & 127 & 164 & 25.4 & 189 & 13.6 & 0.9 & 14.6 & 12.1 \\
\hline Left hypertrophy & 66 & 19 & 66 & 33.8 & 13.2 & 47 & 2.8 & 0.6 & 3.8 & 1.8 \\
\hline Chronic left leg ulcer & 247 & 15 & 41 & 224 & 8.2 & 232 & 15.5 & 0.4 & 16.5 & 14.9 \\
\hline Hepatomegaly & 115 & 18 & 92 & 78.6 & 18.4 & 97 & 5.4 & 0.7 & 6.4 & 4.4 \\
\hline F-statistic & 1.21 & 0.88 & 0.6 & 1.48 & 0.6 & 1.33 & 1.96 & 1.87 & 1.96 & 1.62 \\
\hline p-value & 0.19 & 0.67 & 0.97 & 0.04 & 0.97 & 0.1 & 0 & 0 & 0 & 0.01 \\
\hline Significance & NS & NS & NS & * & NS & NS & $* *$ & $* *$ & $* *$ & $*$ \\
\hline
\end{tabular}

TC: total cholesterol; HDL: HDL-cholesterol; LDL: LDL—cholesterol; TRIG: triglycerides; VLDL: Very Low Density Cholesterol; NHDL: Non-HDL—cholesterol: AR: antherogenic ratio; AIP: Antherogenic Index of Plasma; CRI (I\&II): Castelli Ratio (I\&II); Palpitation IRC: Palpitation Irrespective of Underlying Cause. 
Adverse levels of blood lipids constitute a well-established cardiovascular disease risk factor. ${ }^{14}$ Studies in children and young adults conducted by Clarke et al.; ${ }^{15}$ Berenson et al. ${ }^{16}$ and Freedman et al. ${ }^{17}$ have documented tracking of lipid levels over time, and their association with early atherosclerotic lesions. Several cross-sectional and longitudinal studies have described the changes of lipid levels by age during childhood and adolescence $;^{18,19}$ fewer studies have taken pubertal stage into account. The effects of age distribution in the study were notable. Whereas within the lower age categories (11-20 years), total cholesterol and lipid ratios were reduced in both gender, compared to when partic-

Table 6. Clinical detail * hypercholesterolemia Cross-tabulation $($ Cholesterol reference level $=92-180 \mathrm{mg} / \mathrm{dL})$.

\begin{tabular}{|c|c|c|c|c|}
\hline Clinical detail & $\begin{array}{l}\text { Hyperch } \\
\text { Negative }\end{array}$ & $\begin{array}{l}\text { estero } \\
\text { P } \\
\text { (n) }\end{array}$ & $\begin{array}{l}\text { emia } \\
\text { sitive } \\
(\%)\end{array}$ & Total \\
\hline Acute left ventricular failure & 20 & 3 & 2.7 & 23 \\
\hline Routine checkup & 109 & 23 & 20.4 & 132 \\
\hline $\mathrm{CVA}$ & 30 & 3 & 2.7 & 33 \\
\hline HTN & 137 & 34 & 30.1 & 171 \\
\hline $\mathrm{CCF} 2^{\wedge} \mathrm{O}$ HHDX & 33 & 6 & 5.3 & 39 \\
\hline T2 DM & 24 & 4 & 3.5 & 28 \\
\hline HHDx & 31 & 2 & 1.8 & 33 \\
\hline HIV Encephalopathy & 16 & 1 & 0.9 & 17 \\
\hline Stroke & 18 & 4 & 3.5 & 22 \\
\hline Seizure & 5 & 2 & 1.8 & 7 \\
\hline Chronic Liver $\mathrm{dx}$ & 30 & 5 & 4.4 & 35 \\
\hline RVD & 6 & 1 & 0.9 & 7 \\
\hline PUD & 1 & 0 & 0 & 1 \\
\hline CVD & 32 & 5 & 4.4 & 37 \\
\hline CKD & 12 & 1 & 0.9 & 13 \\
\hline Multiple myeloma & 12 & 0 & 0 & 12 \\
\hline Cervical myelopathy & 6 & 1 & 0.9 & 7 \\
\hline Acute decompensation & 4 & 0 & 0 & 4 \\
\hline Lucipunt wart failure & 2 & 1 & 0.9 & 3 \\
\hline DKA & 2 & 0 & 0 & 2 \\
\hline Poorly controlled BP & 16 & 3 & 2.7 & 19 \\
\hline P.VHD & 11 & 2 & 1.8 & 13 \\
\hline FSLP & 6 & 1 & 0.9 & 7 \\
\hline Left foot gangrene $2^{\wedge} 0$ peripheral & 3 & 2 & 1.8 & 5 \\
\hline Recurrent miscarriage & 2 & 0 & 0 & 2 \\
\hline Hemorrhagic CVD & 1 & 1 & 0.9 & 2 \\
\hline Somatization disorder & 2 & 1 & 0.9 & 3 \\
\hline Elevated BP & 11 & 2 & 1.8 & 13 \\
\hline Palpitation IRC & 8 & 3 & 2.7 & 11 \\
\hline Hypercholesterolemia & 1 & 0 & 0 & 1 \\
\hline Decompensated HHF & 4 & 0 & 0 & 4 \\
\hline Left hemispheric & 1 & 1 & 0.9 & 2 \\
\hline Left ventricular hypertrophy & 2 & 0 & 0 & 2 \\
\hline Chronic left leg ulcer & 0 & 1 & 0.9 & 1 \\
\hline Hepatomegaly & 2 & 0 & 0 & 2 \\
\hline Total & 600 & 113 & & 713 \\
\hline
\end{tabular}

*Palpitation IRC: Palpitation Irrespective of Underlying Cause. ipants were much older. This may relate to sexual maturity. Bertrais et al. ${ }^{20}$ investigated the relationships between lipid levels and sexual maturity, independently of age-related differences, with a view to determining possible differences related to sexual maturity across the percentiles of the lipid distributions. They reported that in boys and girls, total cholesterol levels were significantly associated with pubertal stage after controlling for age, thus emphasizing the importance of sexual maturity, even for a given age, for interpreting lipid levels in children. Lipid levels change markedly by pubertal stage, and patterns differ by sex and race. Chronological age ranges widely within a given pubertal stage and is an insensitive indicator of pubertal stage and the related changes in lipid levels. ${ }^{21}$

The importance of the current study, particularly in measuring the risk of hypercholesterolemia as well as other forms of dyslipidemia has been underscored. This risk may be higher within the active age period of $30-60$ years. The study also significantly demonstrated the elevated lipids levels in younger women in the study population than older men.

The major limitation faced was during the recruitment of participants in the study; a lot of the participants were not willing to give consent for their information to be used in the study.

\section{Conclusions}

During sexual development or maturity, plasma lipid levels change drastically by each developmental stage as seen in the current study; and patterns vary by age and sex. Within a given pubertal stage, chronological age can differ greatly, and in these situations, it is an insensitive predictor of the expected status of changing lipid levels. When assessing the screening and diagnostic criteria for the purpose of classifying individuals with elevated blood lipid levels, pubertal or sexual development may perhaps be taken into account.

Table 7. Age category ${ }^{*}$ hypercholesterolemia Cross-tabulation $($ Cholesterol reference level $=92-180 \mathrm{mg} / \mathrm{dL})$.

\begin{tabular}{lcccc}
$\begin{array}{l}\text { Age category } \\
\text { (years) }\end{array}$ & $\begin{array}{c}\text { Hypercholesterolemia } \\
\text { Negative }\end{array}$ & $\begin{array}{c}\text { Positive } \\
\text { (n) }\end{array}$ & (\%) \\
& \multicolumn{4}{c}{ Total } \\
$<11$ & 13 & 4 & 3.5 & 17 \\
$11-20$ & 44 & 4 & 3.5 & 48 \\
\hline $21-30$ & 91 & 14 & 12.4 & 105 \\
$31-40$ & 117 & 21 & 18.6 & 138 \\
\hline $41-50$ & 106 & 27 & 23.9 & 133 \\
$51-60$ & 184 & 29 & 25.7 & 213 \\
\hline $61-70$ & 25 & 11 & 9.7 & 36 \\
$71-80$ & 11 & 1 & 0.9 & 12 \\
\hline $81-90$ & 3 & 1 & 0.9 & 4 \\
$91-100$ & 4 & 0 & 0.0 & 4 \\
\hline$>100$ & 2 & 1 & 0.9 & 3 \\
Total & 600 & 113 & & 713
\end{tabular}


caemia in middle-aged women: a prospective study. Int J Obes Relat Metab Disord 2001;25:646-51.

\section{References}

1. Sun L, Halaihel N, Zhang W, et al. Role of sterol regulatory element-binding protein 1 in regulation of renal lipid metabolism and glomerulosclerosis in diabetes mellitus. J Biol Chem 2002;277:18919-27.

2. Ike SO, Onyema CT. Cardiovascular diseases in Nigeria: What has happened in the past 20 years? Nig J Cardiol 2020;17:216.

3. Idemudia JO, Atoe KA. A pilot assessment of dyslipidaemia and risk of arteriosclerosis among men in Oredo Local Government Area of Edo State. Nigeria J Res Basic Clin Sci 2019;1:243-52.

4. George J, Rapsomaniki E, Pujades-Rodriguez M, et al. How does cardiovascular disease first present in women and men? Incidence of 12 cardiovascular diseases in a contemporary cohort of 1,937,360 people. Circulation 2015;132:1320-8.

5. Shanmugasundaram M, Rough SJ, Alpert JS. Dyslipidemia in the elderly: Should it be treated? Clin Cardiol 2010;33:4-9.

6. Le J, Zhang D, Menees S, et al. "Vascular Age" Is advanced in children with atherosclerosis promoting risk factors. Circ Cardiovasc Imaging 2010;3:8-14.

7. Qi L, Ding X, Tang W, et al. Prevalence and risk factors associated with dyslipidemia in Chongqing, China. Int J Environ Res Public Health 2015;12:13455-65.

8. Jo Y, Okazaki H, Moon YA, Zhao T. Regulation of lipid metabolism and beyond. Int J Endocrinol 2016:5415767.

9. Swiger KJ, Martin SS, Blaha MJ, et al. Narrowing sex differences in lipoprotein cholesterol subclasses following mid-life: The very large database of lipids (VLDL-10B). J Am Heart Assoc 2014;3:e000851.

10. Godsland IF, Wynn V, Crook D, Miller NE. Sex, plasma lipoproteins, and atherosclerosis: prevailing assumptions and outstanding questions. Am Heart J 1987;114:1467-503.

11. Guthrie JR, Ball M, Dudley EC, et al. Impaired fasting gly-
12. Torng P-L, Su T-C, Sung FC, et al. Effects of menopause on intraindividual changes in serum lipids, blood pressure, and body weight-the Chin-Shan Community Cardiovascular Cohort study. Atherosclerosis 2002;161:409-15.

13. Matthews KA, Crawford SL, Chae CU, et al. Are changes in cardiovascular disease risk factors in midlife women due to chronological aging or to the menopausal transition? J Am Coll Cardiol 2009;54:2366-73.

14. Labarthe DR. Epidemiology and prevention of cardiovascular diseases: a global challenge. Aspen; Gaithersburg, MD: 1998.

15. Clarke WR, Schrott HG, Leaverton PE, et al. Tracking of blood lipids and blood pressures in school age children: the Muscatine study. Circulation 1978;58:626-34.

16. Berenson GS, Srinivasan SR, Bao W, et al. Association between multiple cardiovascular risk factors and atherosclerosis in children and young adults. The Bogalusa Heart Study. N Engl J Med 1998;338:1650-6.

17. Freedman DS, Wang YC, Dietz WH, et al. Changes and variability in high levels of low-density lipoprotein cholesterol among children. Pediatrics 2010;126:266-73.

18. Sprecher DL, Morrison JA, Simbartl LA, et al. Lipoprotein and apolipoprotein differences in black and white girls. The National Heart, Lung, and Blood Institute Growth and Health Study. Arch Pediatr Adolesc Med 1997;151:84-90.

19. Dai S, Fulton JE, Harrist RB, et al. Blood lipids in children: age-related patterns and association with body-fat indices: Project HeartBeat! Am J Prev Med 2009;37:S56-64.

20. Bertrais S, Balkau S, Charles MA, et al. Puberty-associated differences in total cholesterol and triglyceride levels according to sex in French children aged 10-13 years. Ann Epidemiol 2000;10:316-23.

21. Eissa MA, Mihalopoulos NL, Holubkov R, et al. Changes in fasting lipids during puberty. J Pediatr 2016;170:199-205. 